\title{
The repulsive nature of naked singularities from the point of view of Quantum Mechanics
}

\author{
D. Batic ${ }^{1,2}$, D. Chin ${ }^{1}$ and M. Nowakowski ${ }^{3}$ \\ ${ }^{1}$ Department of Mathematics, University of West Indies, Kingston 6, Jamaica \\ ${ }^{2}$ Departamento de Matematicas, Universidad de los Andes, \\ Cra.1E No.18A-10, Bogota, Colombia \\ ${ }^{3}$ Departamento de Fisica, Universidad de los Andes, \\ Cra.1E No.18A-10, Bogota, Colombia
}

\begin{abstract}
We use the Dirac equation coupled to a background metric to examine what happens to quantum mechanical observables like the probability density and the radial current in the vicinity of a naked singularity of the Reissner-Nordström type. We find that the wave function of the Dirac particle is regular in the point of the singularity. We show that the probability density is exactly zero at the singularity reflecting quantum-mechanically the repulsive nature of the naked singularity. Furthermore, the surface integral of the radial current over a sphere in the vicinity of the naked singularity turns out to be also zero.
\end{abstract}




\section{INTRODUCTION}

The possible existence of naked singularities in General Relativity (GR) has troubled the physicists and mathematicians over many years, leading to conjecture that such singularities, although not forbidden by GR, will be shielded from view by an event horizon. Such a conjecture is called the cosmic censorship [1] and has been proved only on special conditions [2]. It was a surprise for the physics community, when numerical simulations revealed that naked singularity may arise from gravitational collapse [3]. This fact brought back on the agenda of physicists the question how troublesome are naked singularity really. Sometimes ago it was found that naked singularities are repulsive in the sense that a particle experiences a repulsive force in the vicinity of these exotic objects [4]. Here, we examine the issue from the point of view of Quantum Mechanics. We do so by employing the Dirac equation in a background metric which contains a naked singularity of Reissner-Nordström type. The main result of our investigation is that neither the wave function nor observables constructed out of the wave function contain any type of singularity. It is as if the quantum mechanical particle would not care about the existence of such an exotic object. This is a surprising result which is in contrast to the classical case where the motion of the test particle cannot be continued over the singularity. Such a result may let think that quantum-mechanically naked singularities are less troublesome than previously thought. Furthermore, the numerical value for the probability density at the singularity turns out to be zero which can be interpreted as the quantum-mechanical reflection of the classical repulsive nature of the naked singularity. A result in the very same spirit concerns the surface integral of the current close to the singularity which is also zero.

It is worthwhile to recall the unpleasant features of a naked singularity which seems to allow the existence of closed time-like curves [5]. In the presence of Reissner-Nordström and Reissner-Nordström-deSitter naked singularities this phenomenon gets reflected in radial velocities $v_{r}(r)$ of a massless particle exceeding the speed of light, whenever the radial distance of the particle from the naked singularity is less than a certain critical value [6]. For instance, already in the Reissner-Nordström naked singularity scenario we have $v_{r}>1$ for $r<Q^{2} / 2 M$.

The paper is organized as follows. In section II we will discuss the Dirac equation in curved space-times. Section III introduces shortly the angular and radial solutions of the 
Dirac equation in the Reissner-Nordström metric and the main results. Section IV is devoted to the Klein-Gordon equation in the aforementioned metric and we show that scalar particles behave similarly to Fermions in the vicinity of a naked singularity. In section $\mathrm{V}$ we draw our conclusions.

\section{DIRAC EQUATION IN CURVED SPACE-TIMES}

To implement the gravity effect into the Dirac equation different approaches have been used in the literature. One of them is through the Newman-Penrose formalism [7]. We follow here the procedure of [8], where the Dirac equation is written in the non-canonical form

$$
\begin{aligned}
& {\left[i \Gamma^{\mu}(x) D_{\mu}-m\right] \Psi(x)=0,} \\
& D_{\mu}=\frac{\partial}{\partial x^{\mu}}-i E_{\mu}-i e A_{\mu}, \\
& E_{\mu}=\frac{i}{2} \rho \partial_{\mu} \rho-\frac{i}{16} \operatorname{Tr}\left(\Gamma^{\nu} \nabla_{\mu} \Gamma^{\lambda}\right) \Gamma_{\nu} \Gamma_{\lambda}+\frac{i}{8} \operatorname{Tr}\left(\rho \Gamma_{\mu} \nabla_{\lambda} \Gamma^{\lambda}\right) \rho, \quad \rho=\frac{i}{4 !} \epsilon_{\mu \nu \lambda \sigma} \Gamma^{\mu} \Gamma^{\nu} \Gamma^{\lambda} \Gamma^{\sigma}
\end{aligned}
$$

where $D_{\mu}$ is the so-called spin derivative including also electrodynamics, $\nabla_{\mu}$ is the covariant derivative w.r.t. the Christoffel symbols and the gamma matrices satisfy the anticommutation relations

$$
\left\{\Gamma^{\mu}(x), \Gamma^{\nu}(x)\right\}=2 g^{\mu \nu}(x)
$$

which allow to couple gravity to the Dirac particle. According to [9], for every static spherically symmetric space-time the combination $\Gamma^{\mu} E_{\mu}$ simplifies to

$$
B=\Gamma^{\mu} E_{\mu}=\frac{i}{2} \nabla_{\mu} \Gamma^{\mu}
$$

and the Dirac equation becomes [10]

$$
\left[i \Gamma^{\mu} \frac{\partial}{\partial x^{\mu}}+\frac{i}{2} \nabla_{\mu} \Gamma^{\mu}+e \Gamma^{\mu} A_{\mu}\right] \Psi=m \Psi .
$$

The above formulation relies on a local $U(2,2)$ symmetry which allows a unified description of electrodynamics and general relativity as a classical gauge theory in the framework of the Dirac equation [8]. It is therefore possible to choose a gauge such that

$$
\nabla_{\mu} \Gamma^{\mu}=0
$$


bringing the Dirac equation to its canonical form used elsewhere in the literature [11]. We emphasize that such a gauge fixing is however not necessary in the context of a gauge theory as any choice of gauge fixing is equivalent. We therefore can also keep $B$ non zero which leads to a non canonical choice of the matrices $\Gamma^{\mu}$. The latter are for the Reissner-Nordström line element

given by [10]

$$
d s^{2}=\Delta(r) d t^{2}-\frac{d r^{2}}{\Delta(r)}-r^{2}\left(d \vartheta^{2}+\sin ^{2} \vartheta d \varphi^{2}\right), \quad \Delta(r)=1-\frac{2 M}{r}+\frac{Q^{2}}{r^{2}}
$$

$$
\Gamma^{t}=\frac{1}{\sqrt{\Delta(r)}} \gamma^{t}, \quad \Gamma^{r}=\sqrt{\Delta(r)} \gamma^{r}, \quad \Gamma^{\vartheta}=\gamma^{\vartheta}, \quad \Gamma^{\varphi}=\gamma^{\varphi}
$$

with

$$
\begin{aligned}
\gamma^{t} & =\gamma^{0} \\
\gamma^{r} & =\gamma^{3} \cos \vartheta+\gamma^{1} \sin \vartheta \cos \varphi+\gamma^{2} \sin \vartheta \sin \varphi, \\
\gamma^{\vartheta} & =\frac{1}{r}\left(-\gamma^{3} \sin \vartheta+\gamma^{1} \cos \vartheta \cos \varphi+\gamma^{2} \cos \vartheta \sin \varphi\right), \\
\gamma^{\varphi} & =\frac{1}{r \sin \vartheta}\left(-\gamma^{1} \sin \varphi+\gamma^{2} \cos \varphi\right),
\end{aligned}
$$

where $\gamma^{0}, \cdots, \gamma^{3}$ are the Dirac matrices in Minkowski space-time. The conserved current is

$$
J^{\mu}=\bar{\Psi} \Gamma^{\mu} \Psi, \quad \nabla_{\mu} J^{\mu}=0
$$

with $\bar{\Psi}=\Psi^{\dagger} \gamma^{0}$. This choice of the adjoint spinor might appear strange is however correct and justified by the fact that the scalar product

$$
(\Psi \mid \Phi)=\int_{\mathcal{H}} \bar{\Psi} \Gamma^{\mu} \Phi \nu_{\mu} d \widetilde{\mu}
$$

with vector field $\nu$ normal to a space-like hypersurface $\mathcal{H}$ is equivalent to the scalar product derived in the Newman-Penrose formalism [12] when we integrate over an hypersurface with $t$ constant and choose the above convention for the adjoint spinor. To be specific, in the presence of a Reissner-Nordström-type naked singularity $\left(M^{2}<Q^{2}\right)$ the scalar product on the hypersurface with $t$ constant reduces to

$$
(\Psi \mid \Phi)=\int_{\mathbb{R}^{3}} \Psi^{\dagger} \Phi \frac{d^{3} x}{\sqrt{\Delta(r)}} .
$$

We notice that in this formulation $J^{0}$ can be interpreted as a positive probability density and $J^{i}$ with $i=1,2,3$ as the spatial component of the probability density current. In particular, $\bar{\Psi} \Gamma^{r} \Psi$ is the component of the current in the radial direction. 


\section{SOLUTIONS OF THE DIRAC EQUATION IN A REISSNER-NORDSTRÖM- TYPE NAKED BACKGROUND}

By the separation ansatz

$$
\Psi(t, r, \vartheta, \varphi)=\frac{1}{r \sqrt{S(r)}} e^{-i \omega t}\left(\begin{array}{c}
\Phi_{j k \omega}^{+}(r, \vartheta, \varphi) \\
\Phi_{j k \omega}^{-}(r, \vartheta, \varphi)
\end{array}\right), \quad S(r)=\sqrt{1-\frac{2 M}{r}+\frac{Q^{2}}{r^{2}}}
$$

with $M^{2}<Q^{2}$ the Pauli spinors $\phi_{j k \omega}^{ \pm}$can be written as

$$
\Phi_{j k \omega}^{+}(r, \vartheta, \varphi)=\left(\begin{array}{c}
\chi_{j-\frac{1}{2}}^{k}(\vartheta, \varphi) \phi_{j k \omega, 1}^{+}(r) \\
i \chi_{j+\frac{1}{2}}^{k}(\vartheta, \varphi) \phi_{j k \omega, 2}^{+}(r)
\end{array}\right), \quad \Phi_{j k \omega}^{-}(r, \vartheta, \varphi)=\left(\begin{array}{c}
\chi_{j+\frac{1}{2}}^{k}(\vartheta, \varphi) \phi_{j k \omega, 1}^{-}(r) \\
i \chi_{j-\frac{1}{2}}^{k}(\vartheta, \varphi) \phi_{j k \omega, 2}^{-}(r)
\end{array}\right)
$$

where

$$
\begin{aligned}
& \chi_{j-\frac{1}{2}}^{k}(\vartheta, \varphi)=\sqrt{\frac{j+k}{2 j}} Y_{j-\frac{1}{2}}^{k-\frac{1}{2}}(\vartheta, \varphi)\left(\begin{array}{l}
1 \\
0
\end{array}\right)+\sqrt{\frac{j-k}{2 j}} Y_{j-\frac{1}{2}}^{k+\frac{1}{2}}(\vartheta, \varphi)\left(\begin{array}{l}
0 \\
1
\end{array}\right), \\
& \chi_{j+\frac{1}{2}}^{k}(\vartheta, \varphi)=\sqrt{\frac{j+1-k}{2 j+2}} Y_{j+\frac{1}{2}}^{k-\frac{1}{2}}(\vartheta, \varphi)\left(\begin{array}{l}
1 \\
0
\end{array}\right)-\sqrt{\frac{j+1+k}{2 j+2}} Y_{j+\frac{1}{2}}^{k+\frac{1}{2}}(\vartheta, \varphi)\left(\begin{array}{l}
0 \\
1
\end{array}\right)
\end{aligned}
$$

for $j=\frac{1}{2}, \frac{3}{2}, \cdots$ and $k=-j,-j+1, \cdots, j$ and $Y_{\ell}^{m}$ are the usual spherical harmonics [13]. The differential equation governing the radial part of the spinor is [10]

$$
\frac{d}{d r} \Phi_{j k \omega}^{ \pm}=\left[\left(\begin{array}{cc}
0 & -1 \\
1 & 0
\end{array}\right) \frac{\omega r-e Q}{r S^{2}} \pm\left(\begin{array}{cc}
1 & 0 \\
0 & -1
\end{array}\right) \frac{2 j+1}{2 r S}-\left(\begin{array}{ll}
0 & 1 \\
1 & 0
\end{array}\right) \frac{m}{S}\right] \Phi_{j k \omega}^{ \pm} .
$$

In the vicinity of the naked singularity the radial system takes a simpler form, namely

$$
\frac{d}{d r} \Phi_{j k \omega}^{ \pm}= \pm \alpha_{j}\left(\begin{array}{cc}
1 & 0 \\
0 & -1
\end{array}\right) \Phi_{j k \omega}^{ \pm}+\mathcal{O}(r), \quad \alpha_{j}=\frac{2 j+1}{2|Q|}
$$

with the solutions

$$
\phi_{j k \omega, 1}^{ \pm}(r) \approx e^{ \pm \alpha_{j} r}, \quad \phi_{j k \omega, 2}^{ \pm}(r) \approx e^{\mp \alpha_{j} r} .
$$

The results ensure that the wave functions are regular at the naked singularity. This enables us to calculate the probability density $J^{0}$ in the vicinity of this exotic object. It is a straightforward computation to show that

$$
J^{0}(r, \vartheta, \varphi)=\frac{|\Psi|^{2}}{\sqrt{\Delta}}
$$


Moreover,

$$
J^{0}(0, \vartheta, \varphi)=0
$$

since the wave function is regular at the naked singularity. This result also demonstrates that the observables constructed out of the wave function are regular and finite at the singularity. Secondly, the fact that the probability density is zero at the singularity can be interpreted in the sense that the singularity has a repulsive nature and this conclusion is independent of the sign of the combinations of the two charges $e$ and $Q$. One could say that the particle avoids the vicinity of the naked singularity from the point of Quantum Mechanics. Briefly, we also give the results for the radial component $\bar{\Psi} \Gamma^{r} \Psi$. The Dirac matrix $\Gamma^{r}$ can be conveniently written in the block form

$$
\Gamma^{r}=\sqrt{\Delta}\left(\begin{array}{cc}
0 & M \\
-M & 0
\end{array}\right), \quad M=\left(\begin{array}{cc}
\cos \vartheta & e^{-i \varphi} \sin \vartheta \\
e^{i \varphi} \sin \vartheta & -\cos \vartheta
\end{array}\right) .
$$

This leads immediately to the expression

$$
J_{j k \omega}^{r}=\frac{2}{r^{2}} \operatorname{Re}\left(\Phi_{j k \omega}^{+\dagger} M \Phi_{j k \omega}^{-}\right)
$$

where

$$
\begin{gathered}
\Phi_{j k \omega}^{+^{\dagger}} M \Phi_{j k \omega}^{-}=\phi_{j k \omega, 1}^{+*} \phi_{j k \omega, 1}^{-} \chi_{j-\frac{1}{2}}^{k^{\dagger}} \chi_{j+\frac{1}{2}}^{k} \cos \vartheta+i e^{-i \varphi} \phi_{j k \omega, 1}^{+^{*}} \phi_{j k \omega, 2}^{-}\left|\chi_{j-\frac{1}{2}}^{k}\right|^{2} \sin \vartheta \\
-i e^{i \varphi} \phi_{j k \omega, 2}^{+*} \phi_{j k \omega, 1}^{-}\left|\chi_{j+\frac{1}{2}}^{k}\right|^{2} \sin \vartheta-\phi_{j k \omega, 2}^{+*} \phi_{j k \omega, 2}^{-} \chi_{j+\frac{1}{2}}^{k^{\dagger}} \chi_{j-\frac{1}{2}}^{k} \cos \vartheta
\end{gathered}
$$

Let us now consider a surface integral

$$
\mathcal{S}_{B(R)}=\int_{B(R)} d A \widehat{\mathbf{n}} \cdot \mathbf{J}=\frac{2}{R^{4}} \operatorname{Re} \int d \Omega \Phi_{j k \omega}^{+\dagger} M \Phi_{j k \omega}^{-}
$$

where $S$ is a sphere of fixed radius $R$ around the naked singularity. In general, we have

$$
\int d \Omega \Phi_{j k \omega}^{+\dagger} M \Phi_{j k \omega}^{-}=\frac{k}{2 j(j+1)}\left(\phi_{j k \omega, 1}^{+^{*}} \phi_{j k \omega, 1}^{-}-\phi_{j k \omega, 2}^{+^{*}} \phi_{j k \omega, 2}^{-}\right) .
$$

Specializing now to a tiny region around the singularity with a radius $\epsilon$ we can use the asymptotic wave functions given in (2) to show that

$$
\mathcal{S}_{B(\epsilon)}=0
$$

This result does not hold necessarily if the sphere on which we integrate is far from the singularity. We can interpret $\mathcal{S}=0$ as follows: the number of ingoing and outgoing particles 
per unit time through the surface of the sphere is zero. We think that this again indicates the quantum-mechanical nature of repulsivity of a naked singularity. More intuitively, the particle does not dwell for a long period in the vicinity of the singularity and in particular, it will not be absorbed.

\section{THE KLEIN-GORDON CASE}

It is illustrative to have a glimpse on the wave function of Klein-Gordon particles in the background of a Reissner-Nordström naked singularity. In such a geometry the Klein-Gordon equation

$$
\left(\square^{2}-m^{2}\right) \Phi=0, \quad \square^{2}=\frac{1}{\sqrt{-g}} \partial_{\mu}\left(\sqrt{-g} g^{\mu \nu} \partial_{\nu}\right)
$$

leads to the equation

$$
\frac{1}{S^{2}} \partial_{t t} \Phi-\frac{1}{r^{2}} \partial_{r}\left(r^{2} S^{2} \partial_{r} \Phi\right)-\frac{1}{r^{2} \sin \vartheta} \partial_{\vartheta}\left(\sin \vartheta \partial_{\vartheta} \Phi\right)-\frac{1}{r^{2} \sin ^{2} \vartheta} \partial_{\varphi \varphi} \Phi-m^{2} \Phi=0
$$

which can be separated by means of the ansatz

$$
\Phi(t, r, \vartheta, \varphi)=e^{-i \omega t} R_{\ell k \omega}(r) Y_{\ell}^{k}(\vartheta, \varphi)
$$

where $\ell=0,1,2, \cdots, k=-\ell,-\ell+1, \cdots, \ell$ and $S$ given by (11). In the naked singularity regime the equation governing the radial part of the wave function reads

$$
\frac{d}{d r}\left(r^{2} S^{2} \frac{d}{d r} R_{\ell k \omega}\right)+\left[\frac{\omega^{2} r^{2}}{S^{2}}+m^{2} r^{2}-\ell(\ell+1)\right] R_{\ell k \omega}=0
$$

In the vicinity of the naked singularity the radial equation simplifies as follows

$$
\left[\frac{d^{2}}{d r^{2}}-\frac{\ell(\ell+1)}{Q^{2}}\right] R_{\ell k \omega} \approx 0
$$

with the solutions

$$
R_{j k \omega, 1}(r) \approx e^{\alpha_{\ell} r}, \quad R_{j k \omega, 2}(r) \approx e^{-\alpha_{\ell} r}, \quad \alpha_{\ell}=\frac{\sqrt{\ell(\ell+1)}}{|Q|} .
$$

The above results ensure that the wave functions are regular at the naked singularity. We introduce the inner product [14]

$$
(\Phi \mid \Psi)=i \int_{\Sigma_{t}} d^{3} x n^{\mu} \sqrt{-\gamma}\left(\Phi \nabla_{\mu} \Psi^{*}-\Psi^{*} \nabla_{\mu} \Phi\right)
$$


where $\Sigma_{t}$ is a space-like hypersurface, $\gamma_{i j}$ is the induced metric on $\Sigma_{t}$ and $n^{\mu}$ is a time-like unit vector normal to $\Sigma_{t}$. Moreover,

$$
J_{K G}^{\mu}=-i\left(\Phi \nabla^{\mu} \Phi^{*}-\Phi^{*} \nabla^{\mu} \Phi\right)
$$

is a conserved current and its zero component is positive definite only if restricted to positive frequency solutions of the Klein-Gordon equation (according to the time-like vector field $t$ ). However, in the case of a naked singularity of the Reissner-Nordström type the vector field $t$ is time-like for all $r>0$. It is a straightforward computation to show that

$$
J_{K G}^{0}(r, \vartheta, \varphi)=2 \omega \frac{|\Phi|^{2}}{\Delta} .
$$

As the wave function is regular at the naked singularity, we obtain

$$
J_{K G}^{0}(0, \vartheta, \varphi)=0
$$

Finally, we proceed as we did for the Dirac equation and the radial component of the current is given by the expression

$$
J_{\ell k \omega, K G}^{r}=\frac{2}{r^{2}} \delta(r)\left|Y_{\ell}^{k}\right|^{2} \operatorname{Im}\left(R_{\ell k \omega} \partial_{r} R_{\ell k \omega}^{*}\right), \quad \delta(r)=r^{2}-2 M r+Q^{2} .
$$

We consider again a surface integral

$$
\mathcal{S}_{B(R)}=\int_{B(R)} d A \widehat{\mathbf{n}} \cdot \mathbf{J}=\frac{2}{R^{4}} \delta(R) \operatorname{Im} \int d \Omega\left|Y_{\ell}^{k}\right|^{2} R_{\ell k \omega} \partial_{r} R_{\ell k \omega}^{*}=\frac{8 \pi}{R^{4}} \delta(R) \operatorname{Im}\left(R_{\ell k \omega} \partial_{r} R_{\ell k \omega}^{*}\right)
$$

where $S$ is a sphere of fixed radius $R$ around the naked singularity. With $\epsilon$ a tiny radius around the singularity we can use the asymptotic wave functions given in (5) to proof that

$$
\mathcal{S}_{B(\epsilon)}=0
$$

since in that region the quantity $R_{\ell k \omega} \partial_{r} R_{\ell k \omega}^{*}$ is purely real

$$
R_{\ell k \omega} \partial_{r} R_{\ell k \omega}^{*}=\left|c_{1}\right|^{2} e^{2 \alpha_{\ell} r}-\left|c_{2}\right|^{2} e^{-2 \alpha_{\ell} r}+2 \operatorname{Re}\left(c_{1} c_{2}^{*}\right) .
$$

\section{CONCLUSIONS}

There exists a class of particular quantum effects with background gravity coupled quantum-mechanically to a relativistic particle, say via the Dirac or Klein-Gordon equation. The peculiarity of such effects is their sharp contrast to the results obtained in the 
classical framework. One such effect is the absence of bound states in the Dirac equation coupled to an exterior black hole metric [10, 15] which is in contrast to the classical result of a stable bound orbit. Another such effect of background gravity is discussed in the present work. Classically, the mere existence of a naked singularity poses many problems, among other the fact that classically the motion of the test particle cannot be continued over the singularity. We showed that the situation is less problematic in the quantum mechanical framework. Indeed, the solutions of the Dirac and Klein-Gordon equation coupled to the Reissner-Nordström metric with a naked singularity reveals that the wave-function is regular at the singularity. Furthermore, the probability density at the singularity is exactly zero and the surface integral over the probability current close to the singularity is also vanishing. This demonstrates that observables constructed from the weave function do not get affected by the presence of the singularity and their value can be continued up to the singularity itself. The above findings can be also interpreted as the quantum mechanical manifestation of the repulsive nature of a naked singularity. From the point of view of quantum mechanics the particle prefers not to dwell close to the singularity. In addition results concerning the quantum mechanical current reveal that the number of particles entering a sphere close to the singularity per unit time is equal to the number of particles leaving the sphere per unit time.

In future, it might be a worthwhile undertaking to construct a velocity operator along the lines explained in [16] in order to determine if the expectation value of the velocity operator takes values bigger than one.

[1] S. W. Hawking and G. F. R. Ellis, The large-scale structure of space-time, Cambridge University Press 1973; T. P. Singh "Singularities and Cosmic Censorship", J. Astrophys. Astr. 18, 335, 1997;R. Penrose, "The Question of Cosmic Censorship", J. Astrophys. Astr. 20, 233, $1999 ;$

[2] S. W. Hawking and G. F. R. Ellis, The large-scale structure of space-time, Cambridge University Press 1973; R. M. Geroch and G. T. Horowitz, "Global Structures of Spacetimes" in S. W. Hawking and W. Israel (eds.) General Relativity: An Einstein Centenary Survey, Cambridge University Press, 1979; G. T. Horowitz, "Finding a Statement of Cosmic Censorship", Gen. 
Relativ. Grav. 10, 1057, 1979; A. Krolak, "A proof of the cosmic censorship hypothesis ", Gen. Relativ. Gravit. 15, 99, 1983; R. P. A. C. Newman, "Cosmic censorship and curvature growth", Gen. Relativ. Gravit. 15, 641, 1983; R. P. A. C. Newman, "A theorem of cosmic censorship - A necessary and sufficient condition for future asymptotic predictability", Gen. Relativ. Gravit. 16, 175, 1984; W. Israel, "Does Cosmic Censorship Exist?", Found. Phys. 14, 1049, 1984; R. P. A. C. Newman, "Cosmic Censorship and the Strength of Singularities" in P. G. Bergmann and V. DeSabbata (eds.) Topological Properties and Global Structure of Space-Time, New York: Plenum Press, 1986

[3] P. Yodzis, H. J. Seifert and H. Müller zum Hagen, "On the occurrence of naked singularities in general relativity", Comm. Math. Phys. 34, 135, 1973; F. J. Tipler, "Note on cosmic censorship", Gen. Relativ. Gravit. 17, 499, 1985; D. M. Eardley, "Naked Singularities in Spherical Gravitational Collapse" in B. Carter and J. B. Hartle (eds.), Gravitation and Astrophysics, Cargese 1987, New York: Plenum Press; A. Ori and T. Piran, "Naked Singularities and Other Features of Self-Similar General-Relativistic Collapse", Phys. Rev. D42, 1068, 1990; S. L. Shapiro and S. A. Teukolsky, "Formation of naked singularities: The violation of cosmic censorship", Phys. Rev. Lett. 66, 994, 1991

[4] F. de Felice, "Repulsive Phenomena and Energy Emission in the Field of a Naked Singularity", Astronomy and Astrophysics 34, 15, 1974; R. Bekgamini, G. Denaedo and A. Treves, "Effective potentials near a Kerr-Newman naked singularity", Lett. Nuovo Cimento 11, 183, 1974; M. Calvani and F. de Felice, "Vortical null orbits, repulsive barriers, energy confinement in Kerr metric", Gen. Relativ. Gravit. 9, 889, 1978; F. de Felice , L. Nobili and M. Calvani, "Charged singularities: repulsive effects", J. Phys. A: Math. Gen. 13, 2401, 1980; I. Gaida , H. R. Hollmann and J. M. Stewart, "Classical and quantum analysis of repulsive singularities in four-dimensional extended supergravity", Class. Quantum Grav. 16, 2231, 1999

[5] C. J. S. Clarke and F. de Felice, "Globally non-causal space-times II: naked singularities and curvature conditions", Gen. Rel. and Grav. 16, 139, 1984; P. S. Joshi, Global Aspects in Gravitation and Cosmology, Oxford University Press, 1993

[6] I. Arraut, D. Batic and M. Nowakowski, "Velocity and velocity bounds in static spherically symmetric metrics", arXiv:1005.1107, 2010

[7] E. T. Newman and R. Penrose, "An Approach to Gravitational Radiation by a Method of Spin Coefficients", J. Math. Phys. 3, 566, 1962; S. Chandrasekhar, The Mathematical Theory 
of Black Holes, Oxford University Press, 1992;

[8] F. Finster, "Local U(2,2) Symmetry in Relativistic Quantum Mechanics", J. Math. Phys. 39, 6276, 1998;

[9] F. Finster, J. Smoller, S.-T. Yau "Particle-Like Solutions of the Einstein-Dirac Equations", Phys. Lett. A259, 431, 1999;

[10] F. Finster, J. Smoller, S.-T. Yau "Non-Existence of Time-Periodic Solutions of the Dirac Equation in a Reissner-Nordstrom Black Hole Background ”, J. Math. Phys. 41, 2173, 2000;

[11] S. Chandrasekhar, The Mathematical Theory of Black Holes, Oxford University Press, 1992;

[12] D. Batic and H. Schmid, "The Dirac propagator in the Kerr-Newman metric ", Prog.Theor.Phys. 116, 517, 2006;

[13] B. Thaller, The Dirac Equation, Springer Verlag, 1992;

[14] S. M. Carroll, An Introduction to General Relativity: Spacetime and Geometry, Addison Wesley, 2004;

[15] A. Lasenby, C. Doran, J. Pritchard, A. Caceres and S. Dolan, "Bound states and decay times of fermions in a Schwarzschild black hole background", Phys. Rev. D72, 105014, 2005; M. Winklmeier and O. Yamada, "Spectral analysis of radial Dirac operators in the Kerr-Newman metric and its applications to time-periodic solutions" J. Math. Phys. 47, 102503, 2006; D. Batic and M. Nowakowski, "On the bound states of the Dirac equation in the extreme Kerr metric", Class. Quant. Grav. 25, 225022, 2008

[16] W. Greiner, Relativistic Quantum Mechanics, wave equations, 3rd edition, Springer-Verlag, 1987 